\title{
ASISTENTE DOMÓTICO A DISTANCIA MEDIANTE LA APLICACIÓN DE TÉCNICAS DE RECONOCIMIENTO DE VOZ
}

\author{
WIRELESS DOMOTIC ASSISTANT USING \\ SPEECH RECOGNITION TECHNIQUES
}

Jairo A. Vélez Pérez ${ }^{1}$, José D. Bejarano Berrio ${ }^{2}$, Carlos E. Arrieta Monsalve ${ }^{3}$, Edilson Delgado Trejos ${ }^{4}$

1. Egresado Ingeniería Electrónica, Semillero MIRP - AEyCC. Facultad de Ingenierías, Instituto Tecnológico Metropolitano -ITM Campus Fraternidad, Calle 54A No. 1-30, Medellín, Colombia. E-mail: jairove lez121149@correo.itm.edu.co

2. Egresado Ingeniería Electrónica, Semillero MIRP - AEyCC. Facultad de Ingenierías, Instituto Tecnológi co Metropolitano -ITM Campus Fraternidad, Calle 54A No. 1-30, Medellín, Colombia. E-mail: josebejara no120018@correo.itm.edu.co

3. Estudiante Semillero MIRP - AEyCC. Facultad de Ingenierías, Instituto Tecnológico Metropolitano ITM Campus Fraternidad, Calle 54A No. 1-30, Medellín, Colombia. E-mail: josebejarano120018@correo.itm. edu.co

${ }^{4}$. Investigador Sénior, Grupo de Investigación en Calidad, Metrología y Producción. Facultad de Ciencias Económicas y Administrativas, Instituto Tecnológico Metropolitano-ITM, Campus Robledo, Calle 73 No 76A - 354, Medellín, Colombia Email: edilsondelgado@itm.edu.co

Recibido: 3 Diciembre

Aceptado: 15 Diciembre

*Correspondencia del autor. Instituto Tecnológico Metropolitano -ITM Campus Robledo, Calle 73 No 76A - 354, Medellín, Colombia Email: edilsondelgado@itm.edu.co

\section{RESUMEN}

Este trabajo presenta una propuesta metodológica de bajo costo y con alto margen de novedad para aplicaciones domóticas. Consiste en la vinculación de un ordenador dotado de un sistema de reconocimiento de voz, una placa de desarrollo Arduino Uno r3 y dispositivos electrónicos de uso común en los montajes electrónicos. El sistema permite realizar de forma automática tareas comunes en una vivienda, a fin de proporcionar un mayor comfort al usuario y prestar asistencia a las personas con algún tipo de discapacidad física, mediante la interacción con el sistema usando comandos de voz. Entre las prestaciones actuales del sistema, están el control de velocidades de ventiladores, control on-off de bombillas, programación de alarmas, monitoreo del estado del sistema (batería, procesador, temperatura y voltaje), control de un televisor, entre otras. El sistema está soportado bajo lenguajes como $\mathrm{C} \#$ y $\mathrm{C}++$. El reconocimiento de voz se basa en la libreria Windows Speech, y el código fuente es ejecutado en entornos de desarrollo integrado (IDE) como Visual Studio 2013 y Arduino IDE, además el sistema incluye un sintetizador de voz SAPI6 para realizar la interacción con el usuario, y para mayor transportabilidad del sistema se incluyen las bondades de la comunicación inalámbrica por medio de módulos de radiofrecuencia (RF). La interacción entre el sistema y el usuario se logró en tiempo real, mejorando los resultados obtenidos con otros sistemas basados en la conexión a Internet que induce retardos no deseados.

Palabras claves: Domótica, red inalámbrica, instrumentación electrónica, comando de voz, reconocimiento de voz. 


\begin{abstract}
A low cost methodology with novel components in domotic applications is presented in this paper. We propose to integrate a speech recognition system in a computer with an Arduino Uno r3 type development system and several common use electronic devices. The integrated system allows different routine home duties to be performed automatically, with the aim of offering increased comfort to users and assisting disabled people who use voice commands. Among several system services, it is important to highlight the speed control of fans, on-off control of lights, alarm schedule, state monitoring of the system (battery, processor, temperature and voltage) and television control. The proposed system is supported by $\mathrm{C \#}$ and $\mathrm{C}++$ languages. Speech recognition uses the Windows Speech library, and the source code is executed on Integrated Development Environment (IDE) as Visual Studio 2013 and Arduino IDE. Furthermore, the system includes a SAPI6 speech synthesizer for interaction with the user, and for improved system transportation, with the benefits of wireless communication through a radio frequency $(\mathrm{RF})$ module. The interaction between system and user was tested in real-time, improving the achievements with another system based on Internet connection, which induces non desired delays.
\end{abstract}

Keywords: Domotics, wireless network, electronic instrumentation, voice command, speech recognition.

\section{INTRODUCCIÓN}

Algunas definiciones de domótica encontradas en la literatura, la relacionan con la automatización del hogar haciendo uso de la tecnología de medios electrónicos con el fin de mejorar la calidad de vida de sus ocupantes (1). En (2), se destaca el uso de las tecnologías de la información y la comunicación para realizar el proceso de automatización de viviendas, mencionando los protocolos de comunicación inalámbrica Bluetooth y Zigbee como uno de los más usados. Las características de un sistema domótico incluyen principalmente el uso de dispositivos electrónicos y sistemas informáticos como la telefonía y el internet, facilitando la interacción, manejo a distancia y capacidad de programación. Actualmente, la domótica es un campo que ha ganado mucha fuerza en el control de los aparatos eléctricos del hogar y permite al usuario realizar tareas de automatización usando sistemas de reconocimientos de voz. Un sistema de reconocimiento de voz permite al computador identificar y entender palabras habladas por una persona usando un micrófono o un teléfono (3). Esto significa que un usuario puede hablar con el PC usando un conjunto de comandos e instrucciones pre-programadas y el PC responderá de la misma manera.

Desde 1975 con la aparición del estándar industrial X10 para la comunicación de dispositivos electróni- cos, la cual fue llevada a la vivienda para la interacción automática entre sensores, actuadores y demás dispositivos, permitieron concebir la domótica como un campo de investigación y desarrollo (1). Investigaciones recientes usan diferentes técnicas y lenguajes de programación para conseguir la interacción del usuario con la vivienda. Por ejemplo, el sistema puede basarse en la interacción de un PC cliente controlado por el usuario y un PC servidor donde se aloja un sistema de reconocimiento de voz basado en las librerías de Microsoft Speech (SAPI), el cual permite traducir las peticiones del usuario en un dato simbólico para ser posteriormente procesado por el PC cliente a fin de efectuar la acción solicitada (2). El problema de este trabajo radica en que la configuración de la voz del usuario se debe realizar escogiendo entre tres tipos de voces con tonos, por ende el sistema soló puede ser usado por quien lo configura, además que el procesamiento por ser remoto tiene retardos en la comunicación, debido a las condiciones climáticas y la cobertura a Internet que se posea. Una limitación frecuente en este tipo de trabajos, es que está sujeto a librerías de Microsoft Windows y por ende el sistema soló podría ejecutarse en computadores con este sistema operativo. En (4), se soluciona el problema de compatibilidad de plataformas usando librerías de Java para el procesamiento de voz, consiguiendo con ello compatibilidad en los distintos sistemas operativos. 
Sin embargo, el problema hallado de este trabajo es la elevada capacidad de cómputo que se necesita para realizar el procesamiento, trayendo consigo un aumento en la instalación, dada la compra de equipos de cómputo de alta gama. Para solucionar la dependencia de Intenet. En (5), se utilizan módulos Bluetooth para la comunicación entre los distintos dispositivos que componen la red, donde cada aparato posee sub módulos Bluetooth que son controlados por un módulo principal y este es accionado por el usuario. El problema es que al ser necesarios tantos módulos Bluetooth como dispositivos a controlar, y dado que todos funcionan en la misma banda de frecuencia, la comunicación presenta retardos considerables. En (6), se presenta un sistema de automatización remoto usando un teléfono que no depende de Internet para su uso, pero tiene la principal desventaja que no posee una interfaz de usuario, además de tener que digitar códigos en el teléfono para poder realizar las tareas de automatización, siendo esto un limitante adicional para su uso cuando el usuario posee alguna discapacidad física. En (7), se usan módulos de comunicación inalámbrica del estándar Zigbee para el envío de datos, y una tarjeta de control embebida SUNPLUS SPCE061A para el reconocimiento de voz. Aunque con esto se logra una interacción entre el usuario y el software sin el uso de internet, presenta limitaciones en cuanto a la cantidad de palabras que se pueden usar para el reconocimiento de voz, contando con la poca capacidad de memoria de la tarjeta, además de que la interacción involucra 4 etapas para realizar una acción determinada, incrementando tiempos de procesamiento del sistema.

En este trabajo se presenta un sistema de control domótico por medio de comandos de voz usando técnicas inalámbricas basadas en transmisión de radiofrecuencias para realizar las tareas de automatización básicas de una vivienda, además de que el procesamiento puede realizarse de manera local, permitiendo una capacidad de comunicación en tiempo real entre el sistema de reconocimiento de voz y los módulos de comunicación.

\section{MATERIALES Y MÉTODOS}

En esta sección se describen los materiales usados para la integración del sistema y los preliminares teóricos así como también el método principal abordado para la conformación del sistema general.

\section{II-A. Insumos del Proyecto}

El sistema consiste en una colección de dispositivos electrónicos que en conjunto permiten realizar una aplicación de control de aparatos electrónicos a través de comandos de voz. Los insumos que intervienen en el proyecto son:

- Un computador portátil con sistema operativo Windows.

- Una placa de desarrollo Arduino Uno r3.

- Módulos RF a 433MHz .

- LEDs infrarrojo.

- Tarjetas de relés.

\section{II-B. Preliminares Teóricos}

1) Domótica: En términos generales, la domótica es la tecnología para desarrollar e implementar la automatización de instalaciones en casas o edificios. Los principales objetivos de la domótica son, seguridad, ahorro de energía, comfort y las comunicaciones (8).

2) Redes inalámbricas: Los estándares de comunicación inalámbrica de frecuente uso en domótica son ZigBee, Radio Frecuencia, Infrarrojo, Wifi u otros. A continuación, algunas definiciones comunes:

ZigBee: Estándar diseñado por la Zigbee Alliance, el cual es un conjunto estandarizado de soluciones que pueden ser implementadas por cualquier fabricante. ZigBee está basado en el estándar IEEE 802.15.4 de redes inalámbricas de área personal (wireless personal área Newark, WPAN) y tiene como objetivo las aplicaciones que requieren comunicaciones seguras con baja tasa de envío de datos y maximización de la vida útil de sus baterías (9).

Infrarrojo: El uso de mandos a distancia basados en transmisión por infrarrojos está ampliamente expendido en el mercado residencial para controlar a distancia equipos de audio y vídeo. Los controladores de equipos domésticos basados en la transmisión de ondas en la banda de los infrarrojos tienen las ventajas de comodidad, flexibilidad y admisión de un gran número de aplicaciones (10). 
Radio frecuencia: Este medio de transmisión puede parecer, en principio, idóneo para el control a distancia de los sistemas domóticos, dada la gran flexibilidad que supone su uso. Sin embargo, resulta particularmente sensible a las perturbaciones electromagnéticas producidas tanto por los medios de transmisión como por los equipos domésticos (10).

\section{II-C. Procedimiento Propuesto}

1) Establecimiento del Diccionario de Referencia: Para que el software tenga la capacidad de reconocer las palabras habladas, es necesario predefinir un diccionario de palabras que servirán como comandos de activación para las distintas tareas. El diccionario permite tantas palabras como se requieran. La estructura de un comando por comodidad siempre es iniciada con el nombre del software y es seguida de la orden que se desea. Por ejemplo, para encender una luz basta decir: Charvis enciende la luz $\mathrm{x}$, donde $\mathrm{x}$ es el número de luz o el lugar donde se encuentra.

2) Conformación del Sistema: En la Figura 1 se ilustra el procedimiento que se propone para el asistente domótico. El sistema consiste en la vinculación de un ordenador dotado de un sistema de reconocimiento de voz, una placa de desarrollo Arduino Uno r3 y dispositivos electrónicos de uso común en los montajes electrónicos.

El sistema permite realizar de forma automática tareas comunes en una vivienda, a fin de proporcionar un mayor comfort al usuario y prestar asistencia a las personas con algún tipo de discapacidad física, mediante la interacción con el sistema usando comandos de voz. Está soportado bajo lenguajes como $\mathrm{C \#}$ y $\mathrm{C}++$. $\mathrm{El}$ reconocimiento de voz se basa en la libreria Windows Speech, y el código fuente es ejecutado en entornos de desarrollo integrado (IDE) como Visual Studio 2013 y Arduino IDE. Este sistema incluye un sintetizador de voz SAPI6 para realizar la interacción con el usuario, y para mayor trasnportabilidad del sistema se incluyen las bondades de la comunicación inalámbrica por medio de módulos de radiofrecuencia (RF). La distribución física de los elementos finales de control, ilustrados

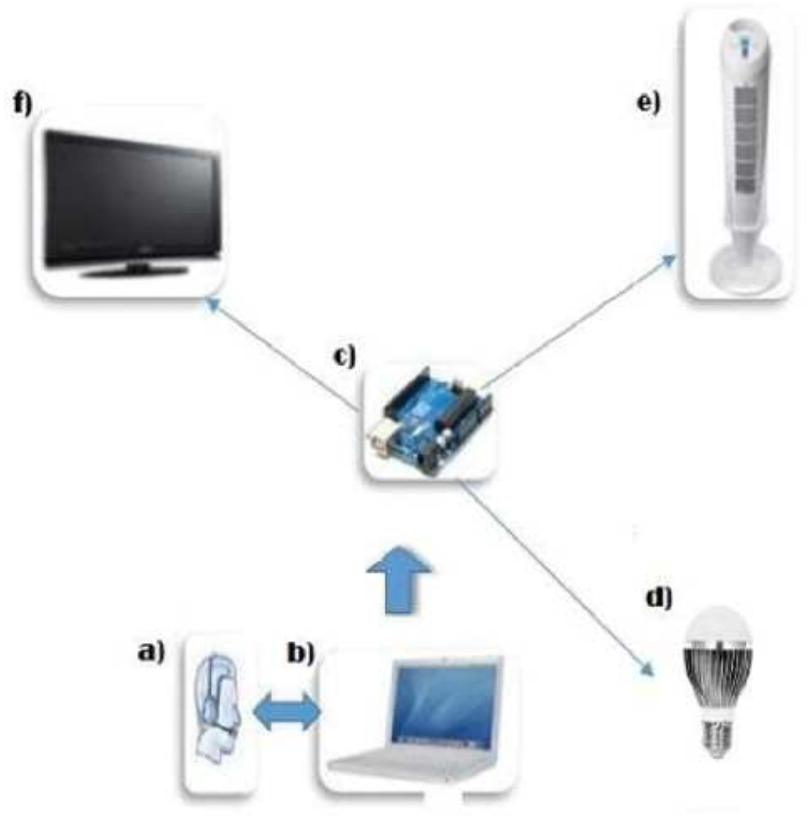

Figura 1: Bloques que conforman el sistema.

en la Figura 1 d), e) y f), respecto al medio de comunicación se representan por la topología estrella, debido a que todos los elementos finales están unidos entre si, a través del controlador principal visto en la Figura 1 c). La arquitectura del sistema es del tipo centralizada ya que todos los elementos están conectados al sistema de control como se muestra en la Figura $1 \mathrm{~b})$. El sistema responde de la siguiente forma: El usuario representado en la Figura 1 a), debe hablar para realizar una petición particular, y la voz es captada por el micrófono del computador y procesada por los algoritmos de programación. Luego, la petición se coteja con las pre-establecidas en el diccionario de referencia, y si corresponde con un comando válido, se realiza la comunicación con la placa Arduino, así como el módulo de radiofrecuencias que se encarga de enviar la orden a otro microcontrolador que ejecuta la acción. Si es para el control del televisor, el microcontrolador envía los datos por infrarrojo al receptor del televisor. Si es el ventilador o el foco de iluminación, el microcontrolador respectivo activa una tarjeta de relevos para encender o apagar las luces, o para cambiar las velocidades del ventilador. El montaje del sistema se muestra en la Figura 2. 


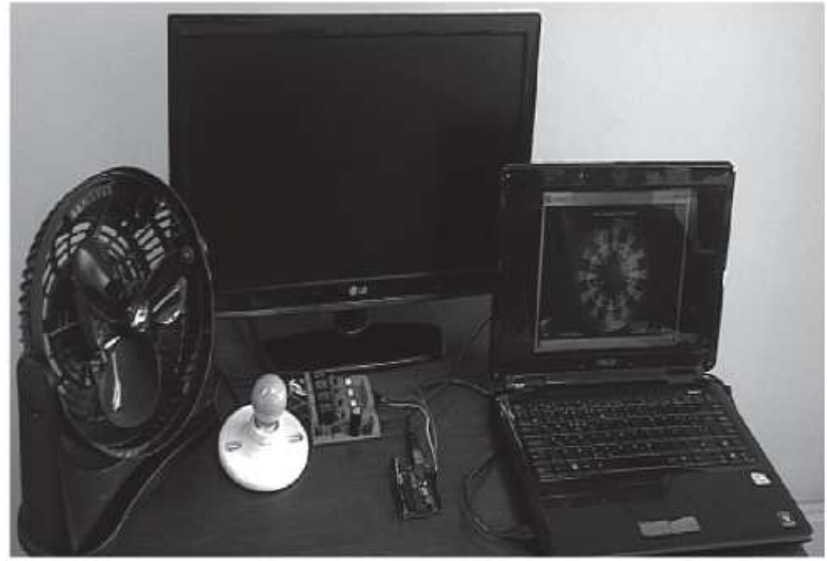

Figura 2: Montaje real del sistema.

\section{RESULTADOS}

Para medir la precisión del sistema de reconocimiento de voz y la velocidad en la ejecución de un comando específico, se realizó la siguiente prueba: Tres personas repitieron 10 veces un mismo comando y se midió el tiempo en milisegundos que el software tarda en dar respuesta a la petición. Por otra parte, la precisión fue medida teniendo en cuenta la cantidad de veces que el sistema era capaz de reconocer un mismo comando. En la Tabla I, se consignan los datos tomados para dos comandos, de donde la línea horizontal (-) representa que el comando no fue procesado. $1 \mathrm{El}$ resumen de tiempos de otros comandos es ilustrado en la Figura $3 a$ y $3 b$. En esta figura se evidencia que los comandos de mayor duración son los que requieren una respuesta hablada de alta duración, caso contrario a los comandos que no dan respuesta hablada y ejecutan la acción de manera inmediata. Los resultados demuestran el buen desempeño del asistente domótico a distancia usando el esquema de implementación propuesto.

Tabla I: Tiempo de respuesta de un comando.

\begin{tabular}{ccc}
\hline & Charvis enciende la luz 1 \\
\hline 1235 & 1303 & 1190 \\
1201 & 1317 & 1188 \\
1191 & 1321 & 1211 \\
1193 & 1175 & 1321 \\
1252 & 1182 & 1348 \\
1320 & 1309 & 1303 \\
1196 & 1228 & 1347 \\
1342 & 1197 & 1347 \\
1314 & 1178 & 1325 \\
1190 & - & 1296 \\
Promedio & & 1259,31 \\
$\%$ precisión & & 96,6 \\
\hline
\end{tabular}

\begin{tabular}{ccc}
\hline \multicolumn{3}{c}{ Charvis enciende el televisor } \\
\hline 1282 & 1406 & 1311 \\
1020 & - & 1296 \\
1739 & 1189 & 1016 \\
1222 & 1712 & - \\
998 & 1213 & 992 \\
1007 & 1014 & 1254 \\
1217 & 1255 & 1292 \\
1200 & 1714 & 1193 \\
- & 1230 & 1301 \\
1713 & 1716 & 1194 \\
Promedio & & 1285,04 \\
$\%$ precisión & & 90 \\
\hline
\end{tabular}

\section{CONCLUSIÓN}

Como resultado del ejercicio técnico-experimental expuesto en este artículo, se logró la implementación de un asistente domótico a distancia a través de la aplicación de técnicas de reconocimiento de voz, usando librerías de Windows Speech, entornos de desarrollo integrado (IDE) como Visual Studio 2013 y Arduino IDE. Todos los algoritmos fueron desarrollados bajo lenguajes $\mathrm{C \#}$ y $\mathrm{C}++$, además de incluir las bondades que ofrecen las redes inalámbricas mediante módulos de radiofrecuencia. El sistema implementado cuenta con la fortaleza de ser fácilmente transportable, además de una rápida reacción a los comandos y poca sensibilidad a las interferencias. Aunque el sistema presenta inmunidad a las perturbaciones acústicas, si en el ambiente hay altos decibelios de ruido, el sistema puede interpretar erróneamente los comandos. En todo caso, el sistema presenta mejorías en su rendimiento cuando los niveles de ruido acústico pueden llevarse a condiciones controladas. Como trabajo futuro, se propone robustecer el diccionario de referencia con el fin de lograr que los comandos puedan ser exitosamente ejecutados, a pesar de que no se digan de una forma estricta. En este sentido, se puede conformar un libro de códigos de actualización inteligente, operando técnicas basadas en sistemas expertos e inteligencia artificial, y optimizando las búsquedas mediante técnicas evolutivas. 


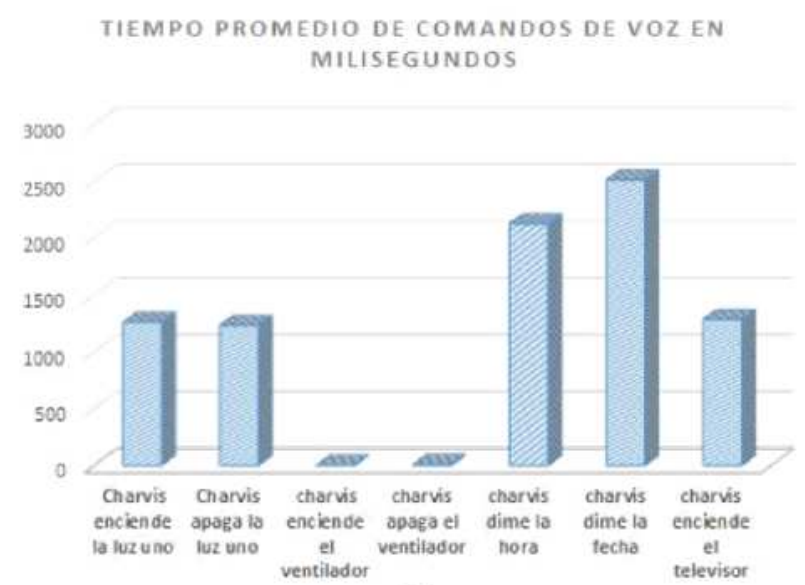

(a)

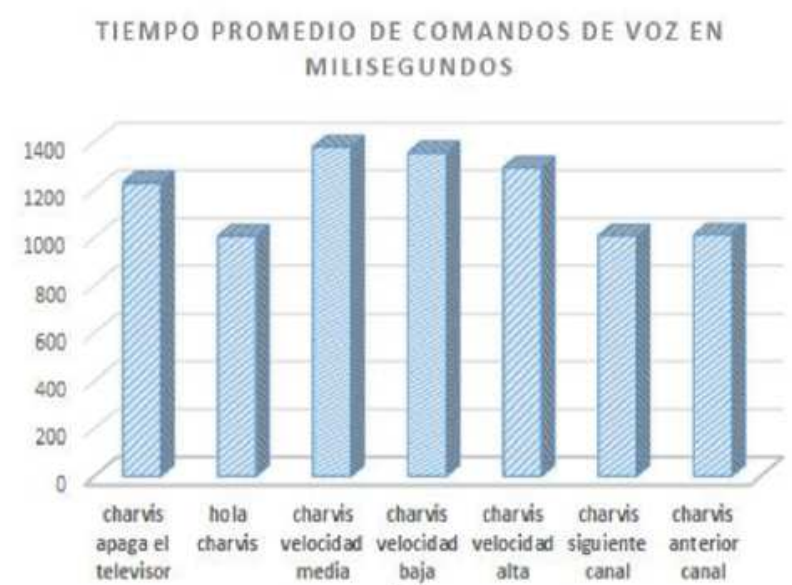

(b)

Figura 3: Diagrama de barras para algunos comandos.

\section{BIBLIOGRAFÍA}

1. K. Gill, S. H. Yang, F. Yao, and X. Lu, “A zigbee-based home automation system,” IEEE Transactions on Consumer Electronics, vol. 55, pp. 422-430, May 2009.

2. S. Jain, A. Vaibhav, and L. Goyal, "Raspberry pi based interactive home automation system." pp. 277-280, Feb. 2014.

3. B. Mardiana et al., "Homes appliances controlled using speech recognition in wireless network environment," in Computer Technology and Development, vol. 2, Nov. 2009, pp. 285-288.

4. A. AL-Ali and M. Al-Rousan, "Java-based home automation system," IEEE Transactions on Consumer Electronics, vol. 50, no. 2, pp. 498-504, 2004.

5. N. Sriskanthan, F. Tan, and A. Karande, "Bluetooth based home automation system," in Microprocessors and Microsystems, vol. 26, no. 6, 2002, pp. 281-289.

6. H. Ardam and I. Coakun, "A remote controller for home and offive appliances by telephone," IEEE Transactions on Consumer Electronics, vol. 44, no. 4, pp. 1291-1297.

7. J. Guo et al., "interactive home-controlled applied to home automation," in Intelligent information Hiding and Multimedia Signal Processing, Sep. 2009, pp. 828-831.

8. F. Mateos et al., "Design and development of an automatic small-scale house for teaching domotics," in Frontiers in Education Conference, vol. 1, 2001, pp. T3C-1.

9. G. D. Goez, A. D. Torres, and E. Delgado, "Estructura de comunicación para el monitoreo y control de un robot geo-posicionado," Master's thesis, Instituto Tecnológico Metropolitano, aug 2011.

10. C. Romero, J. Vasquez, and C. DeCastro, Domótica e inmótica. Viviendas y edificios inteligentes, 2nd ed., RA-Ma, Ed., 2006. 